\title{
Relationship between ankle plantar flexor force steadiness and postural stability on stable and unstable platforms
}

\section{$\operatorname{AUTHOR}(S):$}

Hirono, Tetsuya; Ikezoe, Tome; Taniguchi, Masashi; Yamagata, Momoko; Miyakoshi, Kosuke; Umehara, Jun; Ichihashi, Noriaki

\section{CITATION:}

Hirono, Tetsuya ... [et al]. Relationship between ankle plantar flexor force steadiness and postural stability on stable and unstable platforms. European journal of applied physiology 2020, 120(5): 1075-1082

\section{ISSUE DATE:}

2020-05

URL:

http://hdl.handle.net/2433/263839

\section{RIGHT:}

This is a post-peer-review, pre-copyedit version of an article published in 'European journal of applied physiology'. The final authenticated version is available online at: http://dx.doi.org/10.1007/s00421-020-04346-0.; The full-text file will be made open to the public on 14 March 2021 in accordance with publisher's 'Terms and Conditions for Self-Archiving'.; This is not the published version. Please cite only the published version. この論文は出版社版でありません。引用の際 には出版社版をご確認ご利用ください。 


\section{AUTHOR NAMES and AFFILIATIONS}

5 Tetsuya Hirono ${ }^{1,2}$, Tome Ikezoe ${ }^{1}$, Masashi Taniguchi ${ }^{1}$, Momoko Yamagata $^{1}$, Kosuke Miyakoshi ${ }^{1}$, Jun Umehara ${ }^{1}$

$6 \quad{ }^{2}$, Noriaki Ichihashi ${ }^{1}$

7

8 1. Human Health Sciences, Graduate School of Medicine, Kyoto University, Kyoto, Japan

2. Research Fellow of the Japan Society for the Promotion of Science, Tokyo, Japan

14 Tetsuya Hirono. R.P.T., M.Sc.

15 Human Health Sciences, Graduate School of Medicine, Kyoto University, Kyoto, Japan.

1653 Shogoin-Kawahara-cho, Sakyo-ku, Kyoto 606-8507, Japan

17 TEL : +81-75-751-3967

18 hirono.tetsuya.56x@st.kyoto-u.ac.jp 


\section{ABSTRACT}

\section{Purpose}

This study was aimed at determining the relationship between ankle plantar flexor force steadiness and postural

control during single leg standing on stable and unstable platforms.

\section{Methods}

For the thirty-three healthy participants, force steadiness, at target torques of $5 \%, 20 \%$, and $50 \%$ of the maximum

voluntary torque (MVT) of the ankle plantar flexors, was measured. Force steadiness was calculated as the

coefficient of variation of force. Single leg standing on stable and unstable platforms was performed using the

BIODEX Balance System SD. The standard deviation of the anteroposterior center of pressure (COP)

displacements was measured as the index for postural control. During both measurements, muscle activities of the

soleus were collected using surface electromyography.

\section{Results}

32 On the stable platform, the COP fluctuation significantly correlated with force steadiness at $5 \%$ of MVT $(r=0.512$,

$33 \mathrm{p}=0.002)$. On the unstable platform, the COP fluctuation significantly correlated with force steadiness at $20 \%$ of

34 MVT $(r=0.458, p=0.007)$. However, the extent of muscle activity observed for a single leg standing on both

35 stable and unstable platforms was significantly greater than the muscle activity observed while performing force

steadiness tasks at $5 \%$ and $20 \%$ of MVT, respectively.

\section{Conclusion}

38 Postural stability during single leg standing on stable and unstable platforms may be related to one's ability to 
39 maintain constant torque at $5 \%$ and $20 \%$ of MVT regardless of the muscle activity. These results suggest that the

40 required abilities to control muscle force differ depending on the postural control tasks.

41 
43 force steadiness, force fluctuation, plantar flexor, single leg standing, postural control

44

45

\section{ABBREVIATIONS}

46 COP center of pressure

47 CV coefficient of variation

48 EMG electromyography

49 MVC maximum voluntary contraction

50 MVT maximum voluntary torque

51 RMS root mean square

52 
54 The mechanism for controlling muscle force is important to motor performance. The variability of motor output is generally affected by the variability of a motor unit discharge and environment (Enoka et al. 2003; Moritz et al. 2005). Force steadiness is one aspect of muscle force control. The force fluctuations that occur during submaximal muscle contractions at a target-value torque can be quantified as the force steadiness (Tracy 2007a). The quantification of force steadiness is often obtained during isometric contraction, while torque fluctuation is calculated as the standard deviation, or coefficient of variation (CV), of the amplitude of the torque during a task where submaximal steady contractions must be maintained (Enoka et al. 2003; Oomen and van Dieen 2017; Tracy 2007a). Force steadiness can be affected by the variance in the common synaptic input received by the motor neurons of concurrently active motor units (Feeney et al. 2018; Kornatz et al. 2005; Moritz et al. 2005; Negro et al. 2009). Aging, neurological disorders, and musculoskeletal disorders can also affect force steadiness (Castronovo et al. 2018; Carlyle and Mochizuki 2018; Tracy and Enoka 2002). For example, force steadiness of the knee extensor muscle was significantly more impaired in older adults with knee osteoarthritis than in agematched control individuals (Smith et al. 2014). In a comparison between young adults and older adults, force fluctuations are generally greater in older adults, which indicates a lower force control ability among older adults (Enoka et al. 2003; Kallio et al. 2012; Tracy 2007a). In addition, it has been demonstrated that force fluctuations in older adults with a history of falling were less steady during force matching tasks than that in older adults without any history of falling (Carville et al. 2007). 
coordination of the lower limb muscles by afferents from muscle and tendon of foot (Van Doornik et al. 2011).

concluded that maximum strength of the lower leg muscles was not associated with postural control ability during

quiet standing (Ema et al. 2016). Moreover, a previous study investigating the muscle activities of the extensor

digitorium longus, soleus, peroneus longus, and tibialis anterior during single leg standing on stable and unstable

surfaces revealed that these muscle activities required $10-50 \%$ of the maximum voluntary contraction (MVC)

(Cimadoro et al. 2013). Postural control during standing, therefore, would require an ability to modulate

submaximal muscle torque rather than exert maximum muscle strength.

Among lower limb muscles, the ankle plantar flexor is especially important for postural control,

mobility, and other motor functions (Masani et al. 2003; Stenroth et al. 2015). Regarding age-related changes in

the force steadiness of ankle plantar flexion, it has been reported that, compared with young adults, force

fluctuations of less than 5\% of maximum strength was greater (i.e., unsteadiness) in older adults (Tracy 2007a).

Additionally, the motor unit discharge rate was decreased, and the variability in the motor unit discharge rate was

higher during force steadiness at $10 \%$ and $20 \%$ of the maximum voluntary isometric torque in older adults (Kallio

et al. 2012). Kouzaki and Shinohara (2010) investigated the relationships of ankle dorsiflexor and plantar flexor

force steadiness with COP fluctuation during quiet standing and revealed that anteroposterior COP fluctuation

was significantly positively correlated to plantar flexor force steadiness at $2.5 \%$ and $5 \%$ of maximum strength.

The results suggest that subjects with greater COP fluctuations have less ability to maintain constant muscle force

at low intensity. Furthermore, considering the fact that COP fluctuations during standing could decrease after a 4- 
week training of ankle plantar flexor force steadiness (Oshita and Yano 2011), postural stability during standing

can be especially affected by ankle plantar flexor force steadiness.

(i.e., unstable environments). It is expected that postural control during standing under unstable environment

conditions would require greater muscle force exertion and elaborated COP control using lower limb muscles,

compared to postural control under stable environment conditions (Cimadoro et al. 2013). In fact, approximately

$10 \%$ of the muscle activity needed for maximum ankle plantar flexor strength would be required for controlling

standing posture on a stable platform, whereas approximately $20 \%$ of that same muscle activity would be required

for controlling standing posture on an unstable platform (Cimadoro et al. 2013). Therefore, it would be expected that postural control ability in a stable environment may be related to force steadiness at a relatively low-intensity torque. On the other hand, one's postural control ability in an unstable environment may be related to force steadiness at a greater intensity torque. However, to our knowledge, no study has examined the relationship between ankle plantar flexor force steadiness and postural control ability in unstable environments. Compared to bipedal standing (Kouzaki and Shinohara 2010; Oshita and Yano 2011), single leg standing would be better suited for detecting balance impairments because of its narrow base of support. Additionally, as the same legs were used for single leg standing and force steadiness tasks, the effects of the contralateral leg, in a single leg standing configuration, could be minimized, unlike in bipedal standing; the effects could also clarify the relationship between force steadiness and postural control. Therefore, the purpose of the present study was to investigate the 
110 single leg standing, on both stable and unstable platforms. The hypothesis of the present study was that force

111 steadiness at very low intensity, such as $5 \%$ of maximum voluntary torque (MVT), would be correlated with

112 postural control on stable platforms, and that force steadiness at greater intensity would be correlated with postural

113 control on unstable platforms. 


\section{Participants}

117 Thirty-three young adults (age: $23 \pm 2$ yr., height: $166.6 \pm 8.3 \mathrm{~cm}$, and body mass: $60.3 \pm 11.7 \mathrm{~kg}$ ), including 19

118 men and 14 women, participated in this study. Inclusion criteria required subjects without a history of

119 neuromuscular disorders or surgery on the legs. The purpose and procedures were explained to the participants

before they provided informed written consent to participate in the study. The study was conducted in accordance and Faculty of Medicine (R0548-1).

\section{Procedure}

125 The participants first performed the postural control task, followed by the force steadiness task. For the postural control task, single leg standing with the right leg on either stable or unstable platforms was performed in a random was measured twice. Force steadiness, at intensities of $5 \%, 20 \%$, and $50 \%$ of the MVT of the ankle plantar flexors, was measured twice, in a random order. Force steadiness at 5\% of MVT was found to be related to COP fluctuation during conditions of quiet bipedal standing on a stable platform (Kouzaki and Shinohara 2010), while force steadiness at $20 \%$ of MVT was observed to be related to the sustainable time of quiet standing with a single leg and eyes closed (Oshita and Yano 2010). In addition, postural control during single leg standing in unstable 

of MVT as a high intensity of force steadiness. Therefore, intensities of 5\%, 20\%, and 50\% of MVT for force steadiness tasks were selected in the present study. Muscle activities were also measured using surface electromyography (EMG) during postural control, measuring MVT (i.e., maximum voluntary contraction (MVC) for maximum activation), and force steadiness tasks.

\section{Measurements of postural control tasks}

Postural control tasks consisting of single leg standing were performed using the Biodex Balance System SD

(Biodex Medical Systems, Shirley, NY, USA). Biodex Balance System SD has eight springs located around the perimeter of the balance platform, and the degree of tilt and COP can be measured via these springs. The participants put their bare right foot on the center of the platform and performed a quiet single leg standing for 40

s. Their upper limbs were kept in front their chest, and their hip and right knee angles were kept neutral (i.e., $0^{\circ}$ ), while their left knee was flexed. The participants were instructed to look at a point $30 \mathrm{~cm}$ in front of them while maintaining a natural neck position. They were also instructed as follows: "Please keep your posture upright. When controlling your posture, try to use your ankle joint, with as little hip and knee movement as possible." stable condition, we selected the mode "static" in the Biodex Balance System SD; the platform was not inclined. be inclined about its center, in any direction. The Biodex Balance System SD in the "dynamic" mode varies 
153

154

155

used for the unstable condition (Brown et al. 2018). The COP data acquired during the single leg standing trial were sampled at $20 \mathrm{~Hz}$ and analyzed for $30 \mathrm{~s}$, excluding the first and last $5 \mathrm{~s}$. If a participant touched the platform with their left foot, the trial was repeated under the same conditions.

We focused on the relationship between ankle plantar flexor force steadiness and anteroposterior postural stability, because the muscle function of the ankle plantar flexors could be associated with anteroposterior postural control. Therefore, in this work, the standard deviation of the anteroposterior COP displacements was calculated. In addition, considering the effect of an individual's height and body mass on the center of mass and tilt of the platform, the standard deviation of the COP displacements was divided by their height and body mass, and used as an index of COP fluctuation.

\section{Measurements of maximum voluntary torque and force steadiness}

The tasks of maximum strength and force steadiness were performed using the Biodex System 4 (Biodex Medical

Systems, Shirley, NY, USA). The participants were seated with their hips flexed and their right knee fully extended.

Their trunk, pelvis, and right ankle were fixed with inelastic belts, with their right ankle in a neutral position. The

torque signals obtained from the dynamometer were sent to a personal computer using the software application (MyoResearch XP Master Edition, Noraxon Inc., Scottsdale, Arizona, USA) with a sampling rate of $1500 \mathrm{~Hz}$. The exerted torque was processed with a moving root mean square (RMS) $50 \mathrm{~ms}$ time window in real time because the torque data of plantarflexion via BIODEX included some noise.

The participants were verbally encouraged to exert the maximum ankle plantar flexion for 
approximately $3 \mathrm{~s}$. MVT measurements were performed for two trials with a rest interval of more than one minute.

The averaged peak torques for the two trials were calculated as an individual MVT. Furthermore, the MVT was divided by the individual's body mass $(\mathrm{Nm} / \mathrm{kg})$. Based on the MVT of the ankle plantar flexor, the target torques for the force steadiness tasks were set at 5\%, 20\% and, 50\% of the MVT for individual participants. Each force steadiness task was performed twice, in random order, with a sufficient rest interval. During the force steadiness tasks, the target and exerted torques were shown on the monitor of the personal computer for visual feedback. In a previous study (Tracy 2007a), the time frame for analysis was set to approximately $5 \mathrm{~s}$ for a force steadiness at $50 \%$ of MVT. With this in mind, the present study set as long a time frame as possible for analysis in order to avoid muscle fatigue. The participants were instructed to exert torque for $25 \mathrm{~s}$; this included a duration of $10 \mathrm{~s}$ where the torque was gradually increased from the baseline torque to the target torque and stabilized at this target value. Therefore, the first $10 \mathrm{~s}$ of torque data were omitted to ensure that the readings were steady. The force steadiness was identified as measuring the $\mathrm{CV}$ of force (100 - standard deviation / mean [\%]) using the last $15 \mathrm{~s}$

A low $\mathrm{CV}$ of force value indicated less force oscillation (i.e., an ability to control force exertion to a higher degree).

\section{Electromyography measurements}

Surface EMG of the right soleus muscle was measured with sampling at $1500 \mathrm{~Hz}$ (MyoResearch XP Master

Edition, Noraxon Inc., Scottsdale, Arizona, USA) during postural control tasks and force exertion tasks (MVC 

line between the medial condylis of the femur and the medial malleolus. The raw EMG signals were processed using a bandpass filter between 20 and $500 \mathrm{~Hz}$ (a fourth-order Butterworth filter). A moving RMS window of 50 ms was used, after which the average amplitude of the EMG during the analysis interval was calculated. The MVC of the ankle plantar flexor was performed twice for $3 \mathrm{~s}$, and the averaged EMG values were used in the following analysis. In the postural control tasks, the EMG analysis interval was $30 \mathrm{~s}$, which was the same as the analysis interval for the COP data. In the force steadiness tasks, the EMG analysis interval was $15 \mathrm{~s}$, which was the same as the analysis interval for the force data. The averaged EMG activity of the two measurements for the force steadiness and MVC tasks was used for analysis. Muscle activities during the postural control and force steadiness tasks were normalized using muscle activity during the MVC.

\section{Statistical Analyses}

Statistical analyses were performed using the Statistical Package for the Social Sciences (SPSS version 22.0; IBM

Japan, Inc., Tokyo, Japan). Paired t tests were performed to compare the index of the COP fluctuations between

stable and unstable conditions. Pearson's correlation coefficients were performed to investigate the relationship

of the COP fluctuations in stable and unstable conditions with force steadiness and maximum strength. When a 
210 same between the postural control and the force steadiness tasks. Statistical significance was set at an alpha ( $\alpha)$

211 level of 0.05 .

212 


\section{Postural control tasks and force steadiness tasks}

215 Table 1 shows the index of the COP fluctuation, $\mathrm{CV}$ of force, and maximum isometric strength. The paired $\mathrm{t}$ tests

216 revealed that the index of the COP fluctuation on unstable platforms was significantly less than that of the COP

217 fluctuation on stable platforms $(\mathrm{p}<0.001)$.

\section{Relationship between COP fluctuation and force steadiness or maximum strength}

220 On the stable platform, the index of the COP fluctuations was significantly positively correlated with CV of force only at $5 \%$ of MVT $(r=0.512, p=0.002$, Fig. $1 \mathrm{~A})$. On the other hand, the COP fluctuations on the stable platform were not correlated with CV of force at $20 \%$ of MVT $(r=0.298, p=0.093$, Fig 1 B), $50 \%$ of MVT $(r=-0.044, p$ $=0.808$, Fig $1 \mathrm{C})$, or maximum isometric strength $(\mathrm{r}=-0.298, \mathrm{p}=0.093$, Fig $1 \mathrm{D})$.

$\mathrm{CV}$ of force only at $20 \%$ of MVT $(r=0.458, \mathrm{p}=0.007$, Fig $2 \mathrm{~B})$. On the other hand, the COP fluctuations on the unstable platform were not correlated with $\mathrm{CV}$ of force at $5 \%$ of MVT $(r=0.276, p=0.121$, Fig $2 \mathrm{~A}), 50 \%$ of $\operatorname{MVT}(r=0.331, p=0.060$, Fig $2 \mathrm{C})$, or maximum isometric strength $(r=0.051, p=0.779$, Fig $2 \mathrm{D})$. 
232 the muscle activity observed during a single leg standing on an unstable platform $(33.7 \pm 14.8 \% \mathrm{MVC})$ was

233 significantly greater than the muscle activity observed during the force steadiness task at $20 \%$ of MVT (26.3 \pm

$2349.7 \%$ MVC, $\mathrm{p}=0.001)$ 
237 The current study investigated whether COP fluctuations during single leg standing on stable and unstable

238 platforms are related to ankle plantar flexor force steadiness. We found that the COP fluctuations on a stable

239 platform was correlated with force steadiness only at 5\% of MVT, whereas the COP fluctuations on an unstable

240 platform was correlated with force steadiness only at 20\% of MVT. These results supported our hypothesis that

241 postural control in an unstable environment would be related to force steadiness at a greater intensity when

compared to that in a stable environment. To the best of our knowledge, this is the first study that provides evidence

that anteroposterior COP fluctuations on support surfaces with different stabilities were each related to ankle

plantar flexor force steadiness at different intensities. Interestingly, the intensity of the force steadiness at which

the correlation was observed in the stable condition differed from that in the unstable condition. Our findings

indicate that force steadiness might be affected by different mechanisms based on the difficulty of the motor task

or if neural adaptation interfered with the motor task. If the intensity of the force steadiness related to specific

performance of that task.

less those that on the stable platform. In the stable condition, as the platform was locked and did not tilt,

participants could move their COP over a large area. On the other hand, in the unstable condition, the platform 

as compared to the stable condition. Additionally, this study did not use visual feedback of the COP displacement during postural control tasks. In the unstable condition, the COP displacement is assumed to be affected by additional afferent information, including a sense of equilibrium related to the platform tilt or movement related to the ankle joint change, unlike in the stable condition. Posture was controlled by using the interaction between sensory functions (such as somatosensory, equilibrium, and visual information about the surrounding environment) and motor functions (such as reflex system or voluntary contractions) (Horak 2006).This implies that the $\mathrm{COP}$ in the unstable condition might experience less fluctuation due to additional afferent information. maximum strength and force steadiness at high-intensity contractions but were significantly related to force steadiness only at the low-intensity force of $5 \%$ of MVT. Kouzaki and Shinohara (2010) reported that the COP fluctuations on the stable floor were significantly correlated with force steadiness at $2.5 \%$ and $5 \%$ of MVT. Oshita and Yano (2012) also found that the anteroposterior COP velocity was significantly associated with force

fluctuations during an single leg standing task, which has a smaller area of base support and requires additional muscle activity (Garcia-Masso et al. 2016). Similar to previous studies, our results showed that the COP 
273 might contribute to the control of the COP on a stable surface. Further research will be needed to investigate the

274 contribution of neural adaptation for postural control.

On the unstable platform, the COP fluctuations were not associated with force steadiness at $5 \%$ of MVT,

but significantly correlated at $20 \%$ of MVT. When a greater intensity force steadiness task was performed, the

blood oxygenation level-dependent responses in the ipsilateral parietal lobule, putamen, insula, and contralateral

superior frontal gyrus during isometric contraction increased (Yoon et al. 2014). The responses in the areas were

also associated with force fluctuations. In addition, it is accepted that high-intensity force exertion could apply

high pressure on a participant's sole, from which additional somatosensory could be stimulated. The difficult

postural tasks would require increased innervation from the cerebral cortex, such as supplementary motor area

(Jacobs and Horak 2007; Nandi et al. 2018; Solis-Escalante et al. 2019), additional somatosensory, and

demanded extra regulation from the central nervous system and additional afferent sensory from the peripheral

contractions as $50 \%$ of MVT. 
292 force steadiness task at 20\% of MVT) to determine whether the correlations observed were due to a similar level

293 of muscle activation between two tasks. The results showed that the muscle activity observed during the single

294 leg standing on the stable platform was greater than the muscle activity during the force steadiness task at $5 \%$ of

295 MVT. Moreover, the muscle activity during single leg standing on the unstable platform was also greater than the muscle activity during the force steadiness task at $20 \%$ of MVT. Unexpectedly, even though a significant correlation between COP fluctuations and force steadiness was observed, the degree of muscle activity differed between the two tasks. Some studies (Oomen and van Dieen 2017; Jacobs and Horak 2007; Hunter et al. 2016) reported that both postural control and force steadiness were related to the neuromuscular system. In particular, force steadiness was influenced by a variability of the motor unit discharge rate (Moritz et al. 2005) and muscle afferents, such as muscle spindle and somatosensory (Harwood et al. 2014; Mani et al. 2019). These factors may relationship between postural control and force steadiness. Other muscle neurophysiological behaviors or required to clarify the causes of this relationship from the perspective of the neuromuscular system. COP displacement data during the single leg standing tasks. If the relationship between the muscle activity pattern and COP displacement can be investigated, it is expected that a detailed neuromuscular control system may be realized. Second, five participants who were left-leg dominant (out of thirty-three) were included. The differences 
311 tasks. However, it is possible that this dominant effect was minimized as the analysis of the correlation between

312 force steadiness and postural control was performed using observations obtained from legs of the same dominance.

313 Another limitation was that force steadiness and the EMG of the ankle dorsi flexor were not measured; the tibial

314 anterior muscle can also contribute to postural control (Day et al. 2017). Therefore, future study of dorsi flexion

315 behavior for postural control may be of interest, particularly in unstable conditions. Finally, the participants of this study were limited to healthy young adults. Therefore, it is unclear whether our findings could be applied to populations with impaired postural control or force control, such as older adults and patients with neurological disorders. Further studies are required to clarify the relationship between force steadiness and postural control in older adults or patients with neurological disorders.

320 
In conclusion, we investigated the relationship of the anteroposterior COP fluctuations during single leg standing

323 with ankle plantar flexor force steadiness at $5 \%, 20 \%$, and $50 \%$ of the MVT in healthy young adults. Our results

324 revealed that the COP fluctuations on the stable platform were correlated with force steadiness only at 5\% of MVT.

325 In contrast, the COP fluctuations on the unstable platform were correlated with force steadiness only at $20 \%$ of

326

MVT.

327 
329 This work was supported by a Grant-in-Aid from the Japan Society for the Promotion of Science Fellows

$330 \quad(19 \mathrm{~J} 14772)$.

331 
The authors have no conflicts of interest relevant to this article.

336

of the Kyoto University Graduate School and Faculty of Medicine (R0548-1).

342 The purpose and procedures were explained to the participants before they provided informed written consent to participate in the study.

344 
Brown SR, Brughelli M, Lenetsky S (2018) Profiling Single-Leg Balance by Leg Preference and Position in Rugby Union Athletes. Motor Control 22 (2):183-198. doi:10.1123/mc.2016-0062

Carlyle JK, Mochizuki G (2018) Influence of post-stroke spasticity on EMG-force coupling and force steadiness in biceps brachii. J Electromyogr Kinesiol 38:49-55. doi:10.1016/j.jelekin.2017.11.005

Carville SF, Perry MC, Rutherford OM, Smith IC, Newham DJ (2007) Steadiness of quadriceps contractions in young and older adults with and without a history of falling. Eur J Appl Physiol 100 (5):527-533. doi:10.1007/s00421-006-0245-2

Castronovo AM, Mrachacz-Kersting N, Stevenson AJT, Holobar A, Enoka RM, Farina D (2018) Decrease in force steadiness with aging is associated with increased power of the common but not independent input to motor neurons. J Neurophysiol 120 (4):1616-1624. doi:10.1152/jn.00093.2018

Cimadoro G, Paizis C, Alberti G, Babault N (2013) Effects of different unstable supports on EMG activity and

Day J, Bent LR, Birznieks I, Macefield VG, Cresswell AG (2017) Muscle spindles in human tibialis anterior encode muscle fascicle length changes. J Neurophysiol 117 (4):1489-1498. doi:10.1152/jn.00374.2016

Ema R, Saito M, Ohki S, Takayama H, Yamada Y, Akagi R (2016) Association between rapid force production by the plantar flexors and balance performance in elderly men and women. Age (Dordr) 38 (5-6):475-483. doi:10.1007/s11357-016-9949-3 
contribute to differences in motor performance between young and old adults. Journal of

Feeney DF, Mani D, Enoka RM (2018) Variability in common synaptic input to motor neurons modulates both force steadiness and pegboard time in young and older adults. J Physiol 596 (16):3793-3806. doi:10.1113/JP275658

Fitzpatrick R, Rogers DK, McCloskey DI (1994) Stable human standing with lower-limb muscle afferents providing the only sensory input. J Physiol 480 ( Pt 2):395-403. doi:10.1113/jphysiol.1994.sp020369

Garcia-Masso X, Pellicer-Chenoll M, Gonzalez LM, Toca-Herrera JL (2016) The difficulty of the postural control task affects multi-muscle control during quiet standing. Exp Brain Res 234 (7):1977-1986. doi:10.1007/s00221-016-4602-z

Harwood B, Cornett KM, Edwards DL, Brown RE, Jakobi JM (2014) The effect of tendon vibration on motor unit activity, intermuscular coherence and force steadiness in the elbow flexors of males and females. Acta Physiol (Oxf) 211 (4):597-608. doi:10.1111/apha.12319

Horak FB (2006) Postural orientation and equilibrium: what do we need to know about neural control of balance to prevent falls? Age Ageing 35 Suppl 2:ii7-ii11. doi:10.1093/ageing/afl077

Hunter SK, Pereira HM, Keenan KG (2016) The aging neuromuscular system and motor performance. J Appl Physiol (1985) 121 (4):982-995. doi:10.1152/japplphysiol.00475.2016 
Kallio J, Sogaard K, Avela J, Komi P, Selanne H, Linnamo V (2012) Age-related decreases in motor unit discharge rate and force control during isometric plantar flexion. J Electromyogr Kinesiol 22 (6):983-989. doi:10.1016/j.jelekin.2012.05.009

Kornatz KW, Christou EA, Enoka RM (2005) Practice reduces motor unit discharge variability in a hand muscle and improves manual dexterity in old adults. J Appl Physiol (1985) 98 (6):2072-2080. doi:10.1152/japplphysiol.01149.2004

Kouzaki M, Shinohara M (2010) Steadiness in plantar flexor muscles and its relation to postural sway in young and elderly adults. Muscle Nerve 42 (1):78-87. doi:10.1002/mus.21599

Mani D, Feeney DF, Enoka RM (2019) The modulation of force steadiness by electrical nerve stimulation applied to the wrist extensors differs for young and older adults. Eur J Appl Physiol 119 (1):301-310 doi:10.1007/s00421-018-4025-6

Masani K, Popovic MR, Nakazawa K, Kouzaki M, Nozaki D (2003) Importance of body sway velocity information in controlling ankle extensor activities during quiet stance. J Neurophysiol 90 (6):3774-3782. doi:10.1152/jn.00730.2002

Moritz CT, Barry BK, Pascoe MA, Enoka RM (2005) Discharge rate variability influences the variation in force fluctuations across the working range of a hand muscle. J Neurophysiol 93 (5):2449-2459. doi:10.1152/jn.01122.2004

Nandi T, Fisher BE, Hortobagyi T, Salem GJ (2018) Increasing mediolateral standing sway is associated with increasing corticospinal excitability, and decreasing M1 inhibition and facilitation. Gait Posture 60:135- 
Negro F, Holobar A, Farina D (2009) Fluctuations in isometric muscle force can be described by one linear projection of low-frequency components of motor unit discharge rates. J Physiol 587 (Pt 24):5925-5938. doi:10.1113/jphysiol.2009.178509

Oomen NM, van Dieen JH (2017) Effects of age on force steadiness: A literature review and meta-analysis. Ageing

Oshita K, Yano S (2010) Relationship between Force Fluctuation in the Plantar Flexor and Sustainable Time for Single-leg Standing. Journal of PHYSIOLOGICAL ANTHROPOLOGY 29 (3):89-93.

Oshita K, Yano S (2011) Low-frequency Force Steadiness Practice in Plantar Flexor Muscle Reduces Postural doi:10.2114/jpa2.30.233 standing by young adults. Percept Mot Skills 115 (1):143-152. doi:10.2466/15.26.29.PMS.115.4.143doi:10.1152/jn.2002.88.3.1097 
421

Solis-Escalante T, van der Cruijsen J, de Kam D, van Kordelaar J, Weerdesteyn V, Schouten AC (2019) Cortical dynamics during preparation and execution of reactive balance responses with distinct postural demands. Neuroimage 188:557-571. doi:10.1016/j.neuroimage.2018.12.045

Stenroth L, Sillanpaa E, McPhee JS, Narici MV, Gapeyeva H, Paasuke M, Barnouin Y, Hogrel JY, Butler-Browne G, Bijlsma A, Meskers CG, Maier AB, Finni T, Sipila S (2015) Plantarflexor Muscle-Tendon Properties are Associated With Mobility in Healthy Older Adults. J Gerontol A Biol Sci Med Sci 70 (8):996-1002. doi:10.1093/gerona/glv011

Tracy BL (2007a) Force control is impaired in the ankle plantarflexors of elderly adults. Eur J Appl Physiol 101 (5):629-636. doi:10.1007/s00421-007-0538-0

Tracy BL (2007b) Visuomotor contribution to force variability in the plantarflexor and dorsiflexor muscles. Hum Mov Sci 26 (6):796-807. doi:10.1016/j.humov.2007.07.001

Tracy BL, Enoka RM (2002) Older adults are less steady during submaximal isometric contractions with the knee extensor muscles. J Appl Physiol (1985) 92 (3):1004-1012. doi:10.1152/japplphysiol.00954.2001

Van Doornik J, Azevedo Coste C, Ushiba J, Sinkjaer T (2011) Positive afferent feedback to the human soleus muscle during quiet standing. Muscle Nerve 43 (5):726-732. doi:10.1002/mus.21952

Yoon T, Vanden Noven ML, Nielson KA, Hunter SK (2014) Brain areas associated with force steadiness and intensity during isometric ankle dorsiflexion in men and women. Exp Brain Res 232 (10):3133-3145. doi:10.1007/s00221-014-3976-z 
442 Fig. 1 Relationship between the index of the COP fluctuation on the stable platform, and force steadiness or

443 maximum strength

444 (a) relationship to $\mathrm{CV}$ of force at $5 \%$ of MVT

445 (b) relationship to CV of force at $20 \%$ of MVT

446 (c) relationship to CV of force at $50 \%$ of MVT

447 (d) relationship to maximum isometric strength

448 COP center of pressure; CV coefficient of variation; MVT maximum voluntary torque

449 
450 Fig. 2 Relationship between the index of the COP fluctuation on the unstable platform, and force steadiness or

451 maximum strength

452 (a) relationship to $\mathrm{CV}$ of force at $5 \%$ of $\mathrm{MVT}$

453 (b) relationship to CV of force at $20 \%$ of MVT

454 (c) relationship to CV of force at $50 \%$ of MVT

455 (d) relationship to maximum isometric strength

$456 \mathrm{COP}$; center of pressure, CV; coefficient of variation, MVT; maximum voluntary torque 
Table 1. The indexes of COP fluctuations, $\mathrm{CV}$ of force, and maximum isometric strength.

Postural control tasks

COP fluctuation on a stable platform $\left(\mathrm{cm} /(\mathrm{cm} \cdot \mathrm{kg}) \times 10^{-5}\right) \quad 6.58 \pm 1.90 *$

COP fluctuation on an unstable platform $\left(\mathrm{cm} /(\mathrm{cm} \cdot \mathrm{kg}) \times 10^{-5}\right) \quad 4.82 \pm 1.59 *$

Force steadiness tasks

CV of force at $5 \%$ of MVT (\%)

$1.73 \pm 0.59$

CV of force at $20 \%$ of MVT $(\%)$

$1.34 \pm 0.40$

$\mathrm{CV}$ of force at $50 \%$ of MVT $(\%)$

$1.37 \pm 0.39$

Maximum isometric strength $(\mathrm{Nm} / \mathrm{kg})$

$2.39 \pm 0.41$

* The paired $t$ test revealed a significant difference $(p<0.001)$.

COP: center of pressure, MVT: maximum voluntary torque 
Table 2. Muscle activities of the soleus muscle during each task (\% MVC).

Postural control tasks

On a stable platform

On an unstable platform

Force steadiness tasks

$5 \%$ of MVT

$20 \%$ of MVT

$50 \%$ of $\mathrm{MVT}$
$27.5 \pm 10.4^{\mathrm{a}}$

$33.7 \pm 14.8^{b}$

$26.3 \pm 9.7^{b}$

$51.5 \pm 14.1$

a: A significant difference between the two tasks, which showed a significant correlation between postural control on a stable platform and force steadiness at 5\% MVT.

b: A significant difference between the two tasks, which showed a significant correlation between postural control on an unstable platform and force steadiness at 20\% MVT.

MVT; maximum voluntary torque 

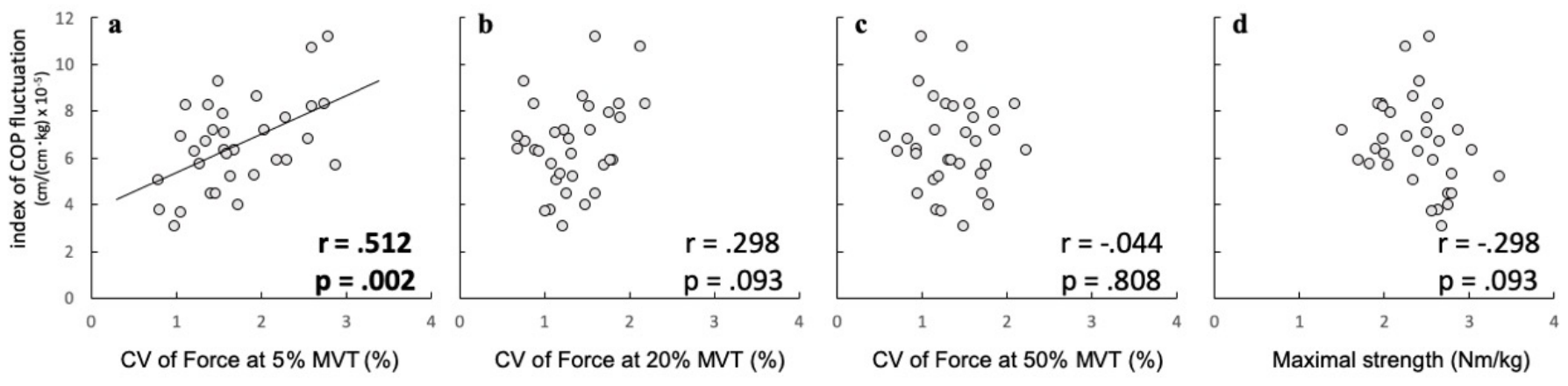

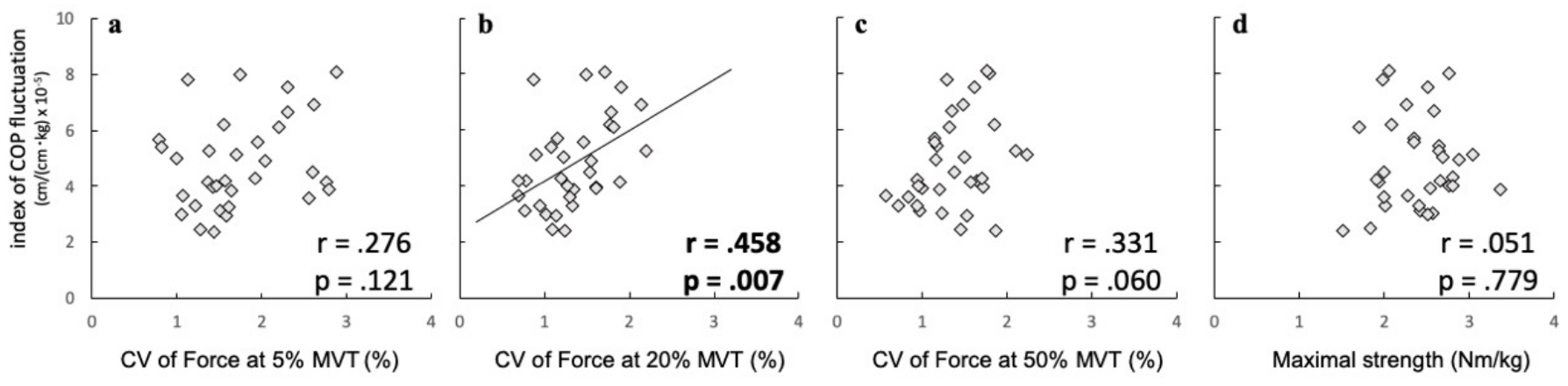